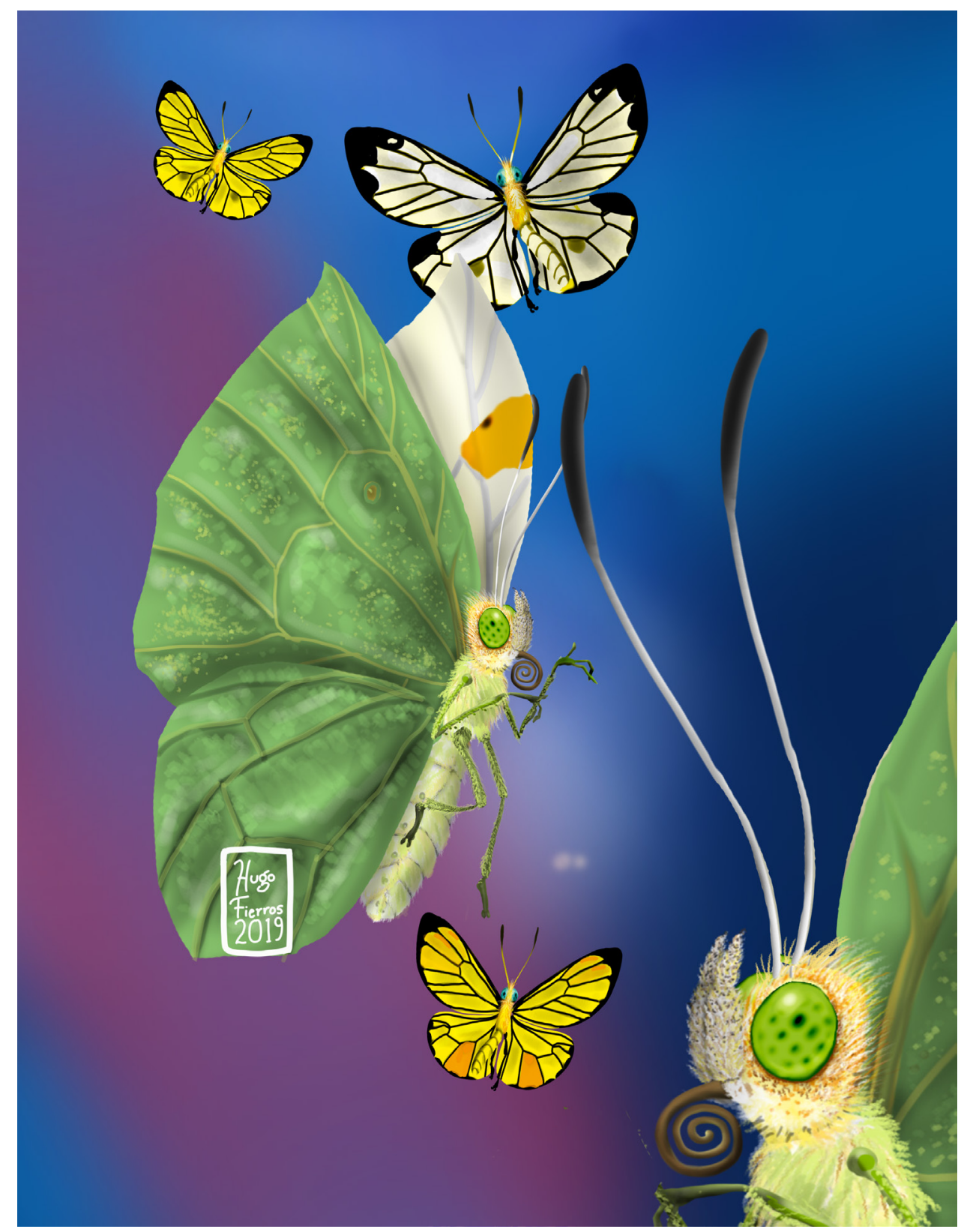

Dugesiana, Año 26, No. 2, julio 2019-diciembre 2019 (segundo semestre de 2019), es una publicación semestral, editada por la Universidad de Guadalajara, a través del Centro de Estudios en Zoología, por el Centro Universitario de Ciencias Biológicas y Agropecuarias. Camino Ramón Padilla Sánchez \# 2100, Nextipac, Zapopan, Jalisco, Tel. 37771150 ext. 33218, http://148.202.248.171/dugesiana/index.php/DUG/index, glenusmx@gmail.com. Editor responsable: José Luis Navarrete Heredia. Reserva de Derechos al Uso Exclusivo 04-2009-062310115100203, ISSN: 2007-9133, otorgados por el Instituto Nacional del Derecho de Autor. Responsable de la última actualización de este número: José Luis Navarrete Heredia, Editor y Ana Laura González-Hernández, Asistente Editorial. Fecha de la última modificación 25 de julio 2019, con un tiraje de un ejemplar.

Las opiniones expresadas por los autores no necesariamente reflejan la postura del editor de la publicación.

Queda estrictamente prohibida la reproducción total o parcial de los contenidos e imágenes de la publicación sin previa autorización de la Universidad de Guadalajara. 
Nota científica

\section{Distribución geográfica de Phanaeus furiosus Bates, 1887 (Coleoptera: Scarabaeidae: Scarabaeinae: Phanaeini) y primeros registros para Zacatecas, México}

\section{Distribution of Phanaeus furiosus Bates, 1887 (Coleoptera: Scarabaeidae: Scarabaeinae: Phanaeini) and first record in Zacatecas, Mexico}

Victor Moctezuma ${ }^{1 *}$ y Guillermo Nogueira ${ }^{2} .{ }^{1}$ Red de Ecoetología, Instituto de Ecología A.C., Carretera antigua a Coatepec 351, El Haya, 91000, Xalapa, Veracruz, México. abadonjvpm@hotmail.com.² gnogalg57@yahoo.com.mx. *Autor de correspondencia.

El grupo de especies Phanaeus tridens incluye a cinco especies cercanamente relacionadas, que se distribuyen en México desde el centro de Sonora sobre la costa del pacífico hacia el sureste, y en América Central desde Guatemala hasta Costa Rica: P. daphnis Harold, 1863; P. eximius Bates, 1887; P. furiosus Bates, 1887; P. nimrod Harold, 1863; y $P$. tridens Laporte-Castelnau, 1840 (Edmonds 1994; Arnaud 2002; Edmonds y Zídek 2012).

Existen algunas poblaciones dentro del grupo $P$. tridens que han sido motivo de confusión, una de ellas localizada en Colima. Edmonds $(1994,2003)$ considera que los ejemplares provenientes de Colima son "representantes inusuales" de $P$. tridens. Sin embargo, no provee una explicación a la disyunción de más de $400 \mathrm{~km}$ que se encuentra entre las poblaciones de Colima y P. tridens de Morelos. Arnaud $(2001,2002)$ designa un neotipo para $P$. tridens y sugiere que la población de Colima corresponde a $P$. furiosus, pues los ejemplares comparten la presencia de una carina pronotal basalmente amplia, fuertemente emarginada apicalmente, carácter ausente en $P$. tridens.

Lizardo et al. (2017) citan a P. furiosus del Estado de México, Morelos y Oaxaca. Sin embargo, estos registros son dudosos: no se ajustan dentro del área típica de distribución de $P$. furiosus, pero concuerdan con la distribución de $P$. daphnis. Edmonds (1994) cita a P. furiosus para Sinaloa, en la localidad de $28 \mathrm{mi}$ NE Concordia. Sin embargo, ésta localidad se ubica en el estado de Durango. Lizardo et al. (2017) confirman la distribución de P. furiosus en Durango. De esta forma, la distribución conocida de P. furiosus corresponde a los estados de Colima, Durango, Guanajuato, Jalisco, Michoacán, Sinaloa, Sonora y Nayarit (Edmonds 1994; Arnaud 2002; Lizardo et al. 2017).

Ya que la distribución de $P$. furiosus ha sido explicada, se reportan los primeros registros de la especie en el estado de Zacatecas (Fig. 1). Los nuevos registros corresponden a localidades del municipio de Momax (que colinda con Jalisco). Estas localidades representan registros atípicos que complementan la distribución de P. furiosus, porque quedan fuera de la distribución potencial modelada por Lizardo et al. (2017), quienes utilizaron 19 capas bioclimáticas para predecir la distribución geográfica de los Phanaeini de México.

Los especímenes estudiados se encuentran depositados en las colecciones Gonzalo Halffter (GHC - Instituto de Ecología A.C., Xalapa, Veracruz, México) y Victor Moctezuma (VMC - Xalapa, Veracruz, México). Material examinado: 2 machos, 2 hembras: "México, Zacatecas, Momax, San Lorenzo. 21 58 '2.72” N, 103'13'42.5” O. 18/ VI/2017. 1959 m. G. Nogueira Col. (VMC)"; 5 machos, 14 hembras: "México, Zacatecas, San Lorenzo (San José) de los Mota. 19-VII-2017. 1750 m. G. Nogueira Col. (GHC)"; 4 machos, 3 hembras: "México, Zacatecas, Momax, San Lorenzo. 18/VI/2017. 1959 m. G. Nogueira Col. (GHC)"; 1 hembra: "México, Zacatecas, Momax, La Manchada. 1974 m. G. Nogueira Col. (GHC).

\section{AGRADECIMIENTOS}

Agradecemos al Dr. Gonzalo Halffter (Instituto de Ecología A.C.), quien amablemente nos permitió revisar los ejemplares de su colección.

\section{LITERATURA CITADA}

Arnaud, P. 2001. Description de nouvelles espèces de Phanaeides. (Col. Scarabaeidae). Besoiro, (6): 2-8.

Arnaud, P. 2002. Phanaeini. Les coléoptères du monde, Vol. 28. Hillside Books, Canterbury, United Kingdom.

Edmonds, W.D. 1994. Revision of Phanaeus MacLeay, a New World genus of Scarabaeinae dung beetles (Coleoptera: Scarabaeidae, Scarabaeinae). Natural History Museum of Los Angeles County, Contributions in Science, (443): 1-105.

Edmonds, W.D. 2003. Tribu Phanaeini. (pp. 58-65). En: Morón, M.A. (Ed.). Atlas de los escarabajos de México. Coleoptera: Lamellicornia. Vol. II Familias Scarabaeidae, Trogidae, Passalidae y Lucanidae. Argania Editio, Barcelona, España.

Edmonds, W.D. y J. Zídek. 2012. Taxonomy of Phanaeus revisited: Revised keys to and comments on species of 
the New World dung beetle genus Phanaeus MacLeay,

1819 (Coleoptera: Scarabaeidae: Scarabaeinae:

Lizardo, V., Escobar, F. y O. Rojas-Soto. 2017. Diversity

Phanaeini). Insecta Mundi, (274): 1-108. and distribution of Phanaeini (Coleoptera: Scarabaeidae: Scarabaeinae) in Mexico. Zootaxa, (4358): 271-294.

Recibido: 1de febrero 2019

Aceptado: 25 de marzo 2019

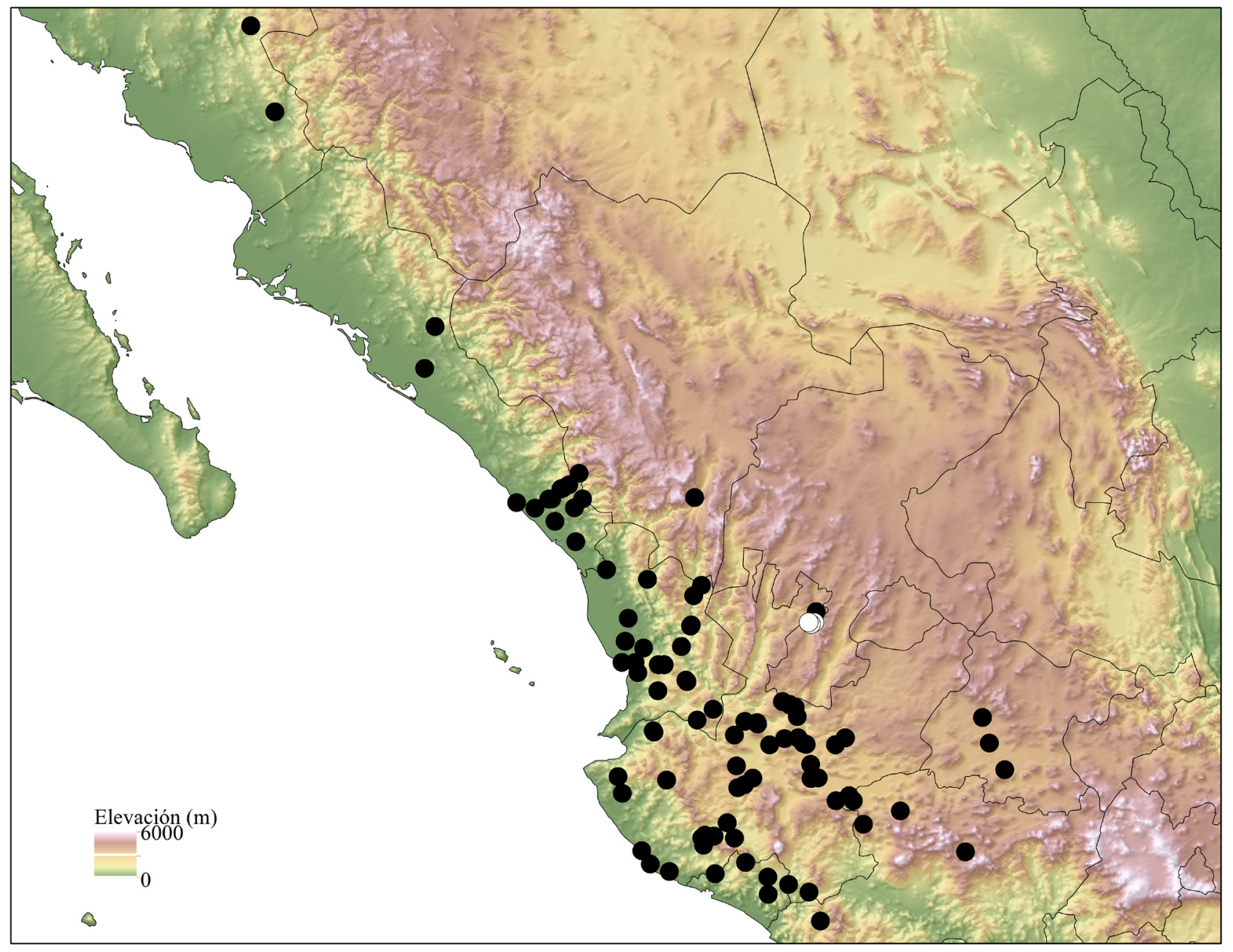

Figura 1. Distribución de P. furiosus: los círculos negros corresponden a los registros anteriores, los círculos blancos corresponden a los nuevos registros. Los registros de distribución fueron tomados de Edmonds (1994) y Lizardo et al. (2017). 